\title{
Mobility and structural constraints of the bonobo trapeziometacarpal joint
}

\author{
TIMO VAN LEEUWEN ${ }^{* *}$, MAARTEN VANNESTE $^{1}$, FAES D. KERKHOF ${ }^{1}$, \\ PRISCILLA D'AGOSTINO ${ }^{1}$, MARIE J. M. VANHOOF ${ }^{1}$, JEROEN M. G. STEVENS ${ }^{2,3}$, \\ G. HARRY VAN LENTHE ${ }^{4}$ and EVIE E. VEREECKE ${ }^{1}$ \\ ${ }^{1}$ Muscles \& Movement, Biomedical Sciences Group, University of Leuven Campus Kulak, Kortrijk, \\ Belgium \\ ${ }^{2}$ Centre for Research and Conservation, Royal Zoological Society Antwerp, Belgium \\ ${ }^{3}$ Behavioural Ecology and Ecophysiology Group, University of Antwerp, Belgium \\ ${ }^{4}$ Biomechanics Section, Department of Mechanical Engineering, University of Leuven, Leuven, Belgium
}

Received 19 June 2018; revised 20 September 2018; accepted for publication 27 September 2018

\begin{abstract}
The human thumb is specialized for manual tasks as it is no longer typically involved in locomotion. However, members of the genus Pan - the closest extant relatives of modern humans - also have a highly mobile thumb, which allows complex manual tasks such as tool-crafting and use. Here, we investigate the thumb kinematics of bonobos (Pan paniscus) in relation to the morphology of their trapeziometacarpal joint using unembalmed bonobo specimens and compare that with the human condition. We use computed tomography-based models of skeletal elements of the thumb during positions of maximum abduction/adduction and flexion/extension to determine the kinematics of the first metacarpal in bonobos and healthy human volunteers. In addition, the 3D geometry of the trapeziometacarpal joint is quantified and, together with an assessment of the ligaments surrounding the joint, is related to the obtained kinematics of the first metacarpal. Our results show a similar trapezial 3D morphology and similar kinematics of the first metacarpal in bonobos and humans, with a markedly higher extension of the first metacarpal in humans. This study provides an integrated analysis of thumb anatomy and kinematics in a unique sample of bonobo specimens.
\end{abstract}

ADDITIONAL KEYWORDS: Pan paniscus - primate anatomy - thumb, thumb ligaments, trapeziometcarpal joint.

\section{INTRODUCTION}

Due to its frequent interaction with the environment, the structure of the hand reflects adaptations related to its use and the level of dexterity of the animal. The thumb is a key component of the hand, with a less mobile thumb having more stability to withstand forces associated with locomotion, whereas a more mobile thumb allows better manipulation and grasping skills (Marzke et al., 2010). The human thumb is a prime example of a highly mobile structure capable of excellent manual dexterity, but several nonhuman primate species possess a similarly mobile thumb and display advanced manipulative capabilities (Christel, 1993; Colell et al., 1995; Christel et al., 1998; Spinozzi et al., 2004; Breuer et al., 2005; Gumert et al., 2011). Members of the genus Pan, the closest

*Corresponding author. E-mail: timo.vanleeuwen@kuleuven.be extant relatives of humans, express a range of complex grip types, such as forceful power grips, in-hand manipulations and dextrous precision grips, as well as thumb opposition, similar to humans (Marzke, 1997; Butterworth \& Itakura, 1998; Christel et al., 1998; Pouydebat et al., 2009; Feix et al., 2015; Bardo et al., 2016). Moreover, like humans, bonobos (Pan paniscus) and chimpanzees (Pan troglodytes) are capable of tool use, a feature that requires relatively complex thumb utilization (Jordan, 1982; Toth et al., 1993; Takeshita \& Walraven, 1996; Ingmanson, 1998; Neufuss et al., 2017). Yet, unlike humans, bonobos and chimpanzees also use their hands during both arboreal and terrestrial locomotion. During terrestrial knuckle-walking, however, the thumb is not typically engaged with the substrate because of its relatively short length (Tuttle, 1967; Wunderlich \& Jungers, 2009). How bonobos and chimpanzees use their thumb during arboreal locomotion is not well documented. Recently, behavioural 
observations by Neufuss et al. (2017) showed that the thumb is important during vertical climbing in wild chimpanzees (and mountain gorillas) but lab-based pressure measurements during vertical climbing in captive bonobos indicated that the pressure beneath the thumb is minimal (Samuel et al., 2018). Such use patterns indicate that the bonobo (and chimpanzee) thumb may be used more for manipulation than previously thought, and might therefore show adaptive signals towards thumb mobility converging to that of the human thumb (Marzke et al., 2015).

The mobility of the thumb and the degree to which it can serve as a prehensile organ is determined by a set of morphological parameters: the position of the trapezium in the carpal complex (which determines the orientation of the distal trapezial joint facet and thus the orientation of the thumb relative to the fingers), the length of the thumb (relative to finger length), the thumb musculature, the trapeziometacarpal joint ligaments, the 3D geometry of the metacarpophalangeal (MCP), interphalangeal (IP) and, in particular, the trapeziometacarpal (TMC) joint (Napier, 1952, 1955, 1960; Rose, 1992). The morphology of the TMC or basal thumb joint has been associated with the range of motion of the thumb and, as a result, with grip capability in different primate species (Rose, 1992). In hominids, the TMC joint is saddle-shaped (Marzke, 1971; Lewis, 1977; Tocheri et al., 2003, 2005; Marzke et al., 2010), the physiological equivalent of a universal joint with two degrees of freedom (Cooney \& Chao, 1977). The majority of movement is guided along the two principal directions of movement of the thumb, namely abduction-adduction and flexion-extension, and is passively constrained particularly by the ligaments crossing the TMC joint, stabilizing the first metacarpal (MC1).

As the modern human's closest extant relatives, members of the genus Pan are essential for interpreting the fossil record and have thus been incorporated in many studies of fossil hominin and hominoid morphology (Rafferty, 1990; Tocheri et al., 2008; Marzke et al., 2010; Almécija et al., 2012; Feix et al., 2015; Skinner et al., 2015; Marchi et al., 2017). However, relatively little is known about the morphology and functional capacity of the thumb in either chimpanzees and, in particular, bonobos. In a recent study (van Leeuwen et al., 2018), we used a series of bonobo cadavers to document and quantify extrinsic and intrinsic hand musculature. The anatomical data indicated a similar muscle configuration and force-generating capacity at the level of the TMC joint in bonobos and humans, but TMC ligament configuration and $3 \mathrm{D}$ joint geometry were not included in the study. The human TMC joint ligament configuration has been studied in detail and comprises a dorsal ligament complex [dorsal radial ligament (DRL), dorsal central ligament (DCL) and posterior oblique ligament (POL)] that reinforces the TMC against dorsal subluxing forces that are associated with power grips and pinches, volar ligaments [anterior oblique ligament (AOL) and ulnar collateral ligament (UCL)] that stabilize the thumb during extension and prevent palmar subluxation, and ulnar ligaments [intermetacarpal ligament (IML) and deep intermetacarpal ligament (DIML)] that stabilize the MC1 (Bettinger et al., 1999; Ladd et al., 2012; D'Agostino et al., 2014; Komatsu \& Lubahn, 2018). In comparison, relatively little is known about non-human primate thumb ligament structures [but see Lewis (1977) and Shrewsbury (2003)] and configurations in different primates need to be further studied.

Here, we investigate both the functional capabilities and the morphology of the bonobo TMC joint in comparison with that of humans. The study primarily investigates the kinematics of the bonobo first metacarpal during the principal movements of the thumb, the geometry of the trapezial articular surface that facilitates movement of the thumb, and the surrounding ligaments that passively restrain the TMC joint. These are subsequently compared to the kinematics and morphology of the human thumb and are related to the manual capabilities of the two species. We hypothesize that the bonobo thumb has a large range of motion in both planes (flexion-extension, abduction-adduction), approaching the level of mobility of the human thumb, to allow for the dextrous manual abilities described in the literature (Marzke, 1997; Christel et al., 1998; Butterworth \& Itakura, 1998; Pouydebat et al., 2009; Feix et al., 2015; Bardo et al., 2016). We expect to see this reflected in the geometry of the joint (a saddle-shaped joint comparable to that of humans), as well as in the ligaments, which should be sufficiently developed to provide passive tension when required but which do not impede heavily on the mobility of the thumb.

\section{MATERIAL AND METHODS}

\section{SPECIMEN ACQUISITION}

Cadaver specimens of five adult bonobos were obtained in collaboration with various European zoos (Table 1). One cadaveric human specimen, obtained via the Human Body Donation Program of the University of Leuven, Belgium, was included for comparison of the anatomical features between bonobo and human. The specimens included the entire forearm and hand and were stored at $-18{ }^{\circ} \mathrm{C}$. The bonobo and human specimens have been included in a previous study focusing on extrinsic and intrinsic hand musculature (van Leeuwen et al., 2018). All individuals died of natural causes.

\section{CT SCANNING AND IMAGE SEGMENTATION}

The specimens were scanned by computed tomography (CT) using a 64-slice Discovery HD 750 CT scanner [GE 
Table 1. Cadaveric specimen details

\begin{tabular}{|c|c|c|c|c|c|c|c|}
\hline Code & $\begin{array}{l}\text { Previous } \\
\text { code* }\end{array}$ & $\begin{array}{l}\text { Subject } \\
\text { identifier }\end{array}$ & Sex & Age (years) & Sample & Origin & Analysis \\
\hline Pp1 & Pp2 & $\begin{array}{l}\text { Zorba† } \\
8365526\end{array}$ & M & Adult 35 & $\mathrm{~L}$ & $\begin{array}{l}\text { Wilhelma Zoo, Stuttgart, } \\
\text { Germany }\end{array}$ & Anatomy/kinematics \\
\hline Pp2 & Pp5 & $\begin{array}{l}\text { Jasiri } \\
15295295\end{array}$ & $\mathrm{~F}$ & Subadult 8 & $\mathrm{~L}$ & $\begin{array}{l}\text { Royal Zoological Society } \\
\text { Antwerp, Belgium }\end{array}$ & Anatomy/kinematics \\
\hline Pp3 & Pp7 & $\begin{array}{l}\text { Ludwig } \\
\text { MIG12- } \\
\quad 29882197\end{array}$ & M & Adult 32 & $\mathrm{R}$ & Zoo Frankfurt, Germany & Anatomy/kinematics \\
\hline $\mathrm{Pp} 4$ & Pp8 & $\begin{array}{l}\text { Kirembo } \\
\text { SB: } 177\end{array}$ & M & Adult 24 & $\mathrm{R}$ & $\begin{array}{l}\text { La Vallée des Singes, Le } \\
\text { Gureau, France }\end{array}$ & Anatomy/kinematics \\
\hline Pp5 & Pp9 & $\begin{array}{l}\text { Hermien } \dagger \\
27641621\end{array}$ & $\mathrm{~F}$ & Adult 39 & $\mathrm{~L}$ & $\begin{array}{l}\text { Wilhelma Zoo, Stuttgart, } \\
\text { Germany }\end{array}$ & Anatomy/kinematics \\
\hline Hs1 & Hs1 & 692 & M & Adult 60 & $\mathrm{~L}$ & $\begin{array}{l}\text { University of Leuven, } \\
\text { Kortrijk, Belgium }\end{array}$ & Anatomy \\
\hline
\end{tabular}

Pp: Pan paniscus, Hs: Homo sapiens, F: female, M: male, L: left arm + hand, R: right arm + hand

* Specimen code in van Leeuwen et al. (2018).

†Wild born.

Healthcare, Little Chalfont, UK; display field of view (DFOV): $250 \mathrm{~mm}$; slice thickness: $0.625 \mathrm{~mm}$; voxel size: $0.15 \mathrm{~mm}^{3} ; 100 \mathrm{kV} ; 180 \mathrm{~mA}$; image size: $512 \times 512$ pixels). Each specimen was scanned in a set of static functional positions including thumb abduction, adduction, flexion and extension, using a custom-designed radiolucent rig according to a protocol adapted from Crisco et al. (2015) and D'Agostino et al. (2017a).

The CT scans were segmented manually using Mimics 19.0 (Mimics for Research 19.0, Materialise, Leuven, Belgium) and the bone elements involved in thumb motion (i.e. the radius, scaphoid, trapezium and MC1) were reconstructed into 3D surface models for each position.

\section{HUMAN VOLUNTEERS}

CT scan data from five healthy human volunteers from a previous study on human thumb kinematics (Kerkhof et al., 2018) were used in our analysis for comparison. Subject details are provided in Table 2. Static $\mathrm{CT}$ scans of the functional positions mentioned above were collected using the same radiolucent rig and with a similar protocol as used to collect the bonobo data. CT-scan segmentation and reconstruction followed the same protocol as used for the bonobo scans.

\section{KINEMATIC ANALYSIS AND 3D JOINT GEOMETRY}

We used custom Matlab (Mathworks, Natick, MA, USA) scripts to register the bonobo and human 3D bone models from each static functional position onto their counterpart (i.e. flexion-extension, abduction-adduction) to determine the kinematics of the bone elements involved
Table 2. Human volunteer details

\begin{tabular}{llllll}
\hline Code & $\begin{array}{l}\text { Subject } \\
\text { identifier }\end{array}$ & Sex & $\begin{array}{l}\text { Age } \\
\text { (years) }\end{array}$ & Sample & Analysis \\
\hline Hs2 & Patient 10 & F & 30 & R & Kinematics \\
Hs3 & Patient 11 & F & 25 & L & Kinematics \\
Hs4 & Patient 12 & F & 37 & R & Kinematics \\
Hs5 & Patient 18 & F & 55 & R & Kinematics \\
Hs6 & Patient 19 & M & 26 & R & Kinematics \\
\hline
\end{tabular}

F: female, M: male, R: right arm + hand, L: left arm + hand.

during thumb flexion and abduction. An iterative closest point (ICP) algorithm was applied to calculate the transformation matrix (i.e. rotation and translation) between the bone models of each investigated thumb motion by minimizing the squared distances between model vertices (Bergström et al., 2011; Bergström \& Edlund, 2017; D'Agostino et al., 2017a). Movements of the MC1 during thumb motion are expressed in a local coordinate system, relative to the trapezium, while the movements of the scaphoid and trapezium are expressed in a radius-based coordinate system.

The local coordinate system is represented by the principal axes of the TMC joint (Fig. 1). As the trapezium articulates with the MC1 through a saddleshaped joint surface, the two principal directions of the saddle represent the two principal movements of the thumb, adduction-abduction and flexion-extension. A fifth-degree polynomial was fitted to the joint surface of the trapezium to determine the saddle point (i.e. where the slope in both principal directions is zero, see below) and the principal directions of curvature, which 


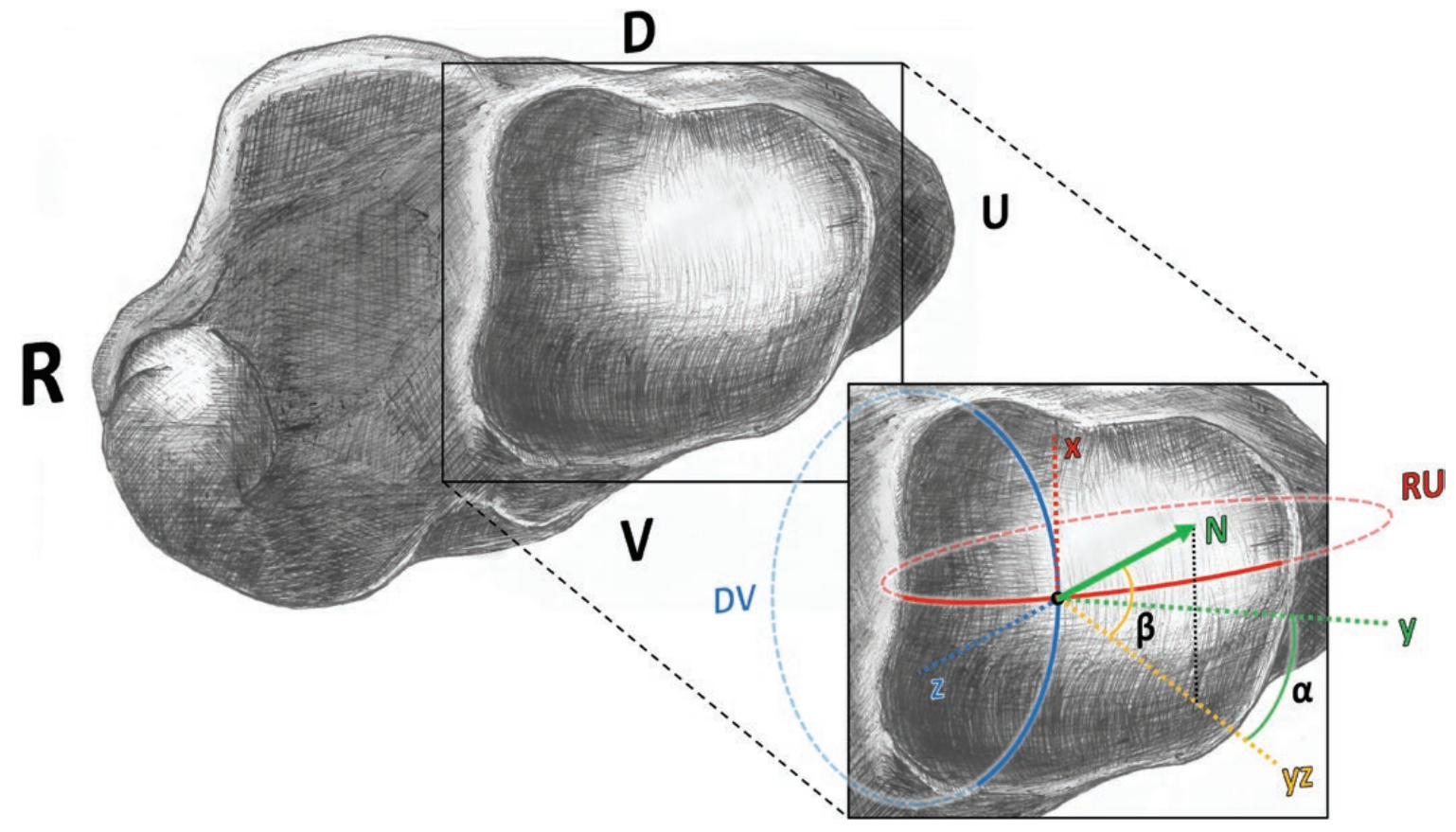

Figure 1. The trapezium coordinate system depicted on a bonobo left hand trapezium, where R, U, D and V are the trapezium's radial, ulnar, dorsal and volar sides, respectively. The local coordinate system is defined by DV, the dorsal-volar, N, the proximal-distal, and RU, the radial-ulnar axis. Rotation along DV results in thumb abduction or adduction, along RU in flexion or extension, and along $\mathrm{N}$ in rotation of the MC1. Joint surface curvature is quantified as the radius that describes the circles DV and RU that describe the curvature in each of the trapezium's principal directions of movement. $\mathrm{N}$ is defined by the saddle point's normal, its relative deviation to the radial coordinate system, here defined by $x$ (radial-ulnar), $y$ (proximal-distal) and $z$ (dorsal-volar), and describes the orientation of the trapezium's distal facet. This deviation is a product of the radial deviation, defined by the azimuth $(\alpha)$, and the volar deviation, defined by the elevation $(\beta)$.

represent the two orthogonal axes of the joint surface that define the coordinate system (Halilaj et al., 2013). The radii of curvature of a 25 -point grid in a 2.25 -mm radius around the saddle point were averaged in each principal direction of the coordinate system, corresponding to the functional axes of the trapezium, to represent joint surface curvature of each direction.

Movements of the trapezium and scaphoid are expressed relative to a radius-based coordinate system, which is defined using manual point registration at the radiocarpal joint using the radial styloid process and ulnar notch as landmarks (Orr et al., 2010; D'Agostino et al., 2017a). The coordinate system is in line with the International Society of Biomechanics convention (Wu \& Cavanagh, 1995; Wu et al., 2005), with dorso-ventral being the $x$-axis, proximo-distal the $y$-axis and radio-ulnar the $z$-axis. Each bone element is fitted onto its counterpart within the same principal motion of the thumb using the ICP algorithm as described above. By using the radius as an anchor point, the rotation and translation of each bone element relative to the radius are determined for thumb flexion and abduction. Subsequently, by subtracting the movements of the previous element in the chain
(radius-scaphoid-trapezium-MC1), movements of each element with respect to the previous element are calculated.

In addition, the trapezium-based coordinate system was used relative to the radius-based coordinate system to determine the orientation of the trapezium within the hand. The saddle point of the trapezium is determined within the radius-based coordinate system, standardized to the radial styloid, to represent the location of the trapezium. The trapezium-based coordinate system's normal, perpendicular to the distal trapezial articular surface, and the proximodistal axis of the radius were aligned. The trapezium's offset from the proximodistal axis was determined and described as angles in both the mediolateral plane, azimuth, and the dorsoventral plane, elevation (Fig. 1). Given the small sample size, we evaluated potential differences between bonobos and humans visually using scatter-dot plots.

\section{DISSECTION OF THE TMC JOINT LIGAMENTS}

After removal of the muscle tissue (van Leeuwen et al., 2018), the TMC ligament structure was dissected according to the technique of Berger (2001), 
and ligament origin and insertion were identified and photographed in situ. Subsequently, the individual ligaments were extracted and documented using a remote-controlled digital camera set-up.

\section{RESULTS}

KINEMATICS OF THE FIRST METACARPAL DURING THUMB ABDUCTION AND FLEXION

Kinematic analysis of the MC1 range of motion with respect to the trapezium during thumb abduction and flexion indicates that bonobos are capable of similar ranges of MC1 motion as humans during thumb abduction, while the human range of MC1 motion during thumb flexion far exceeds that of bonobos, with the exception of one individual (Fig. 2). The mean range of motion of the MC1 in bonobos about the dorsoventral $(x)$ axis during thumb abduction is $31.4 \pm 6.5^{\circ}$, similar to the observed human range of motion of $33.0 \pm 4.5^{\circ}$. In contrast, during thumb flexion, the range of motion of the MC1 about the mediolateral $(z)$ axis in bonobos is $9.5 \pm 2.9^{\circ}$ (after exclusion of outlier Pp2 with a range of $\mathrm{MC} 1$ motion of $53.0^{\circ}$ ) while that of humans is
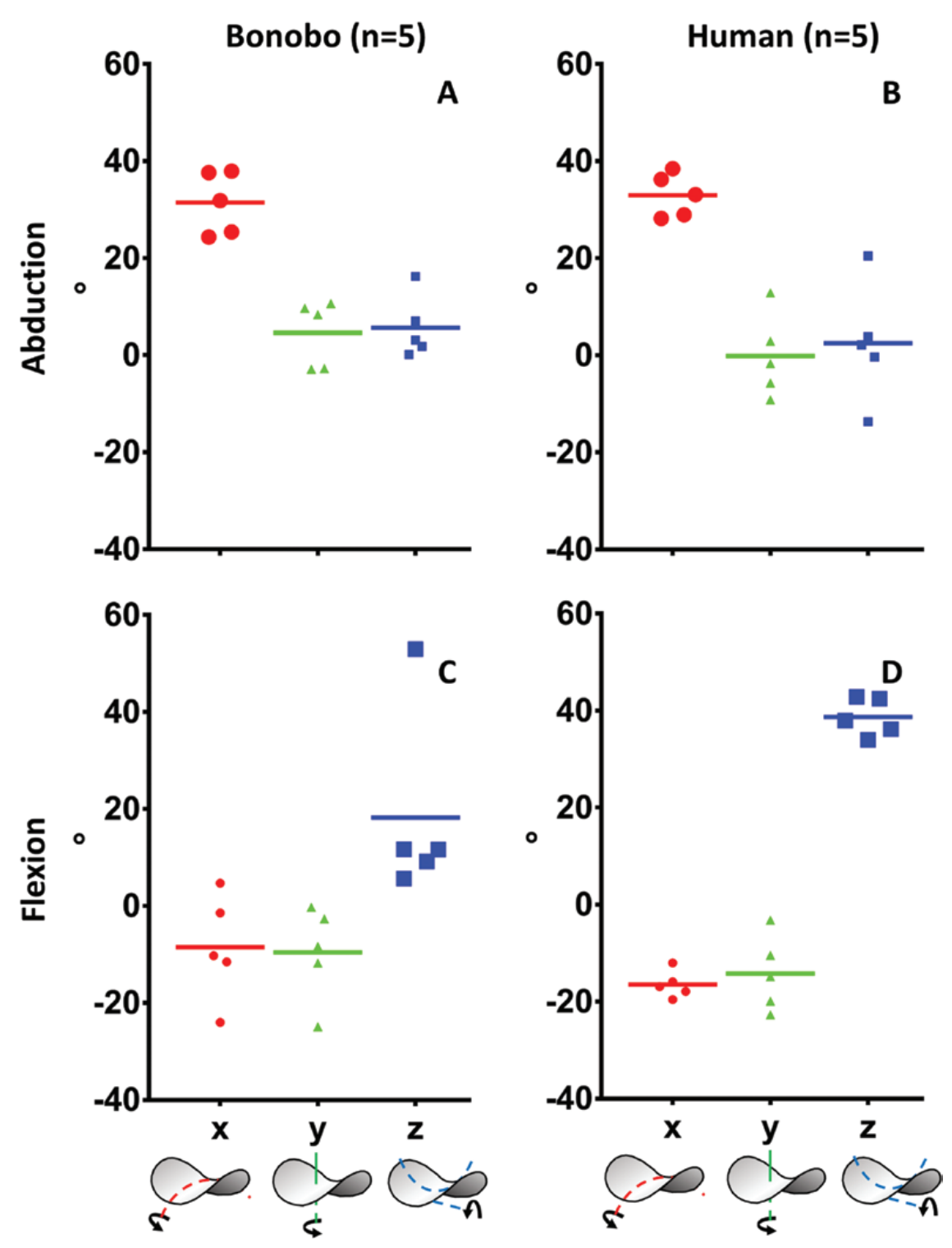

Figure 2. Thumb range of motion represented by rotational angles about the principal axes of the trapezium $(x=$ dorsalvolar, $y=$ proximal-distal, and $z=$ radial-ulnar), during thumb abduction (A, B) and thumb flexion-extension (C, D), in bonobos $(N=5)(\mathrm{A}, \mathrm{C})$ and humans $(N=5)(\mathrm{B}, \mathrm{D})$. Positive rotations about $x$ reflect MC1 abduction, positive rotations about $y$ reflect $\mathrm{MC1}$ exo-rotation, and positive rotations about $z$ reflect $\mathrm{MC} 1$ flexion. 
$38.7 \pm 3.9^{\circ}$. Visual evaluation of the maximum thumb positions indicates that the substantial difference in flexion-extension range of motion between bonobos and humans is due by the higher extension capability of the human MC1 (Fig. 3). Furthermore, the analysis shows that in addition to MC1 rotation about the principal axis during either thumb abduction or flexion, rotation along the other two axes occurs as well. That is, during thumb flexion, $\mathrm{MC} 1$ flexion is combined with abduction and external rotation of $\mathrm{MC} 1$, while during thumb abduction, rotation about the other two axes is more variable, such that either MC1 flexion or extension may occur with either internal or external rotation of the MC1 (Fig. 2).

\section{CURVATURE OF THE DiSTAL ARTICULAR FACET OF THE TRAPEZIUM}

The curvature of the distal articular facet of the trapezium (Fig. 4) is similar between humans and bonobos in the dorsoventral plane, with a convex radius of curvature of $4.1 \pm 1.2 \mathrm{~mm}$ in bonobos and $4.3 \pm 0.8 \mathrm{~mm}$ in humans. However, visual inspection of the curvature along the entire facet shows that the radius of curvature at the level of the saddle point does not describe the curvature of the entire distal facet in the direction of thumb flexion/extension. Instead, the dorsoventral profile of the trapezium's joint surface appears to level off at the most dorsal and most volar aspects of the facet in both bonobos and humans, causing its profile to resemble more of a bell curve than a circle. In contrast, the joint surface curvature of the entire facet in the mediolateral plane is shown to be well represented by the radius of curvature at the saddle point, in both bonobos and humans. In this direction of thumb abduction/adduction, we do see a difference in curvature between bonobos and humans, where the former has a concave radius of curvature of $7.1 \pm 1.5 \mathrm{~mm}$ and the latter $11.1 \pm 1.0 \mathrm{~mm}$.

\section{ORIENTATION AND POSITION OF THE TRAPEZIUM RELATIVE TO THE RADIAL STYLOID}

No clear distinction can made in orientation and/ or position of the trapezium in the carpal complex between bonobos and humans (Fig. 5). The saddle point of the bonobo trapezium is located $11.2 \pm 5.7 \mathrm{~mm}$ lateral, $19.5 \pm 4.1 \mathrm{~mm}$ distal and $6.7 \pm 2.2 \mathrm{~mm}$ volar relative to the radial styloid process. Similarly, the human's trapezial saddle point is located $8.7 \pm 4.3 \mathrm{~mm}$ lateral, $17.7 \pm 1.4 \mathrm{~mm}$ distal and $6.7 \pm 2.4 \mathrm{~mm}$ volar to the styloid. Likewise, the orientation of both species' trapezial articular surfaces is similar, with the normal to the trapezial saddle point facing volarly and radially. Radial deviation from the proximodistal axis of the forearm (azimuth) is $33.9 \pm 9.2^{\circ}$ in bonobos and $39.4 \pm 1.3^{\circ}$ in humans, while deviation towards the volar side of the hand (elevation) is $26.0 \pm 22.3^{\circ}$ in bonobos and $20.5 \pm 13.2^{\circ}$ in humans.

\section{FUNCTIONAL ANATOMY OF THE TMC JOINT LIGAMENTS}

From our observations on the TMC ligament configurations (Fig. 6), we conclude that our single human specimen does conform to the standard descriptions of the TMC ligaments (Bettinger et al., 1999; D'Agostino et al., 2014). We observe a prominent DRL, originating from the trapezium's dorso-radial tubercle and inserting on the dorsal edge of the MC1 base. The POL originates just medially of the DRL, from the dorso-ulnar aspect of the trapezium and inserts onto the dorsoulnar side of $\mathrm{MC} 1$ and towards the palmar-ulnar tubercle. As reported in the literature, the AOL originates

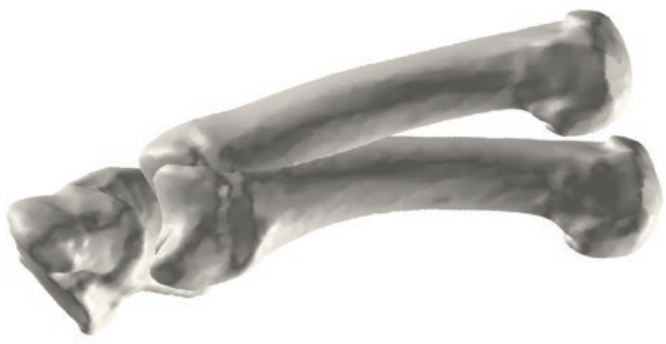

Bonobo

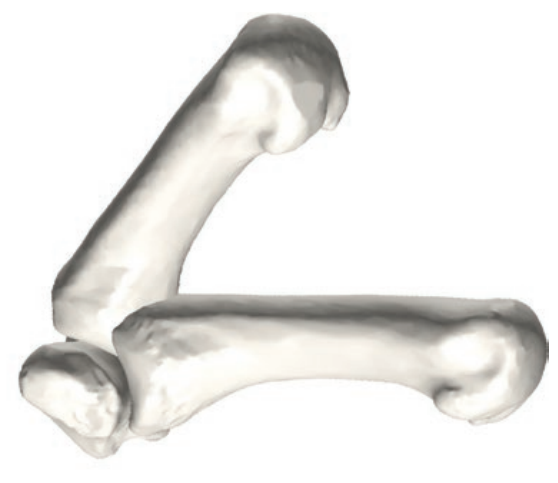

Human

Figure 3. Example of maximal MC1 extension in one bonobo specimen (Pp5) and a human volunteer (Hs4). Note the markedly larger MC1 extension in the human subject. 


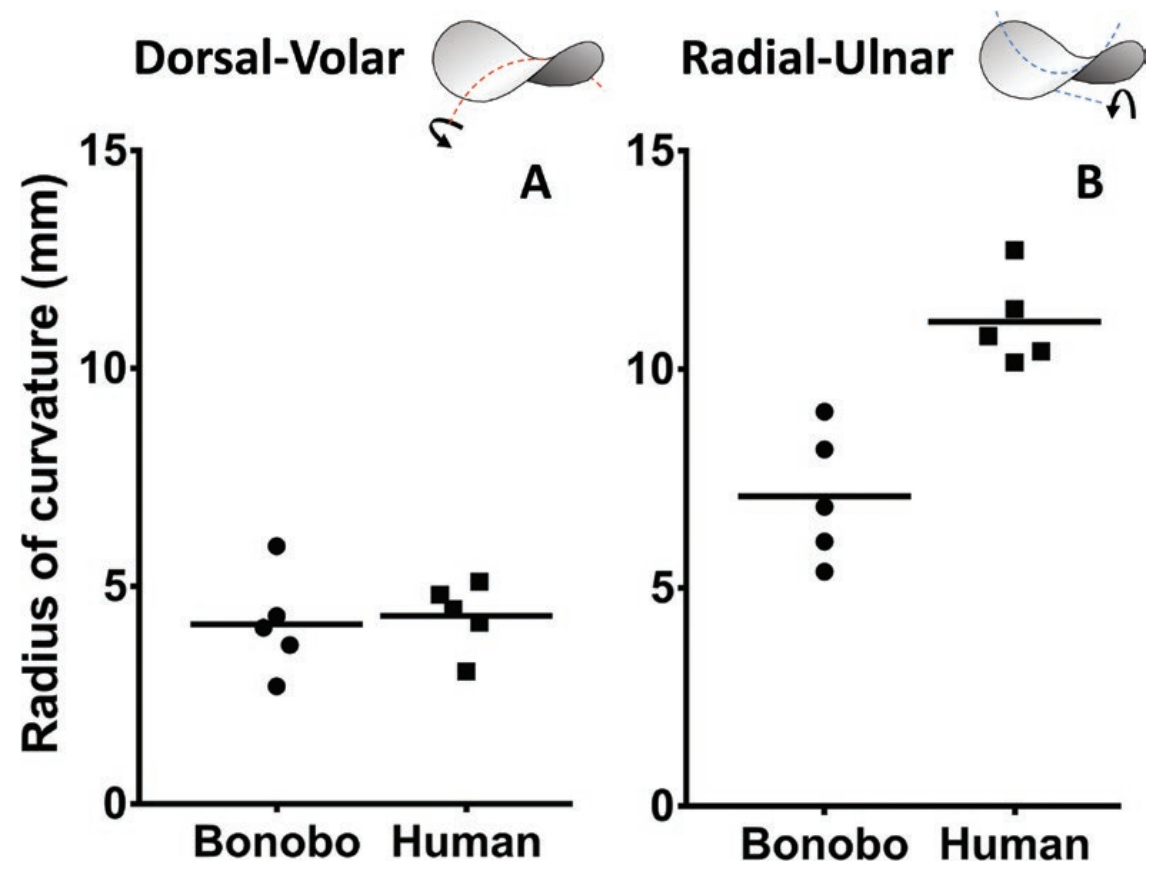

Figure 4. Radii of curvature that describe the circle that lies tangential to the curvature in the trapezium principal directions of movement, flexion (A) and abduction (B), in both bonobos and humans. A larger radius describes a larger circle, and thus a less curved surface.

from the volar tubercle of the trapezium and inserts onto the volar beak of MC1. The AOL in our specimen is translucent, frail and under-developed compared to the dorsal ligaments, which is consistent with previous reports (Bettinger et al., 1999; D'Agostino et al., 2014). The IML, which that stabilizes the MC1, runs from the base of MC2 to the base of MC1, corresponding to the descriptions in the literature (Bettinger et al., 1999; D'Agostino et al., 2014).

In bonobos, the DRL is thin compared to the human DRL, but with a similar origin and insertion. The DRL is characterized by the clearly distinguishable direction of its collagen fibre; in bonobos, however, these fibres are more widely spaced than in humans, adding to its thin appearance. The POL configuration in bonobos is similar to that of humans but relatively well developed when compared to the adjacent DRL. In contrast to the human configuration, the AOL is more prominent in bonobos, compared to the other TMC ligaments and compared to the human AOL. Its origin and insertion are the same as in humans, but it is a thick, non-translucent structure. The IML in bonobos is similar to that of humans in its origin, insertion and dimensions.

\section{DISCUSSION}

Our results show that the anatomy and kinematics of the bonobo thumb display many similarities to the human thumb, as expected given the close phylogenetic relationship between the two species. However, we observe differences in mechanical capabilities, articular surface geometry and ligament configuration, which might be related to dissimilar daily use of the thumb and overall dexterity.

The kinematic analysis shows that bonobos display a smaller range of MC1 motion during thumb flexion than during thumb abduction. Moreover, the range of motion of the MC1 during thumb flexion is markedly lower than observed in humans, displaying a limitation of close to $30^{\circ}$, which may lead to more restricted thumb mobility. Evaluation of the positions of maximum flexion and extension in both species indicates that the larger range of motion of the human MC1 in the dorsoventral plane can be attributed to humans' capacity to further extend the MC1 compared to bonobos (Fig. 3). The orientation of the trapezium does not explain this higher extension potential of the human MC1 given that the position and orientation of the trapezium in the carpal complex is similar between bonobos and humans. Also, surface curvature analysis shows no specific differences between bonobos and humans that could explain a larger range of motion of the MC1 during thumb flexion (i.e. the dorsoventral radius of curvature is similar in the bonobo and human trapezium). However, dissections of the ligaments surrounding the TMC joint do show relatively well-developed volar ligaments in bonobos. The human 

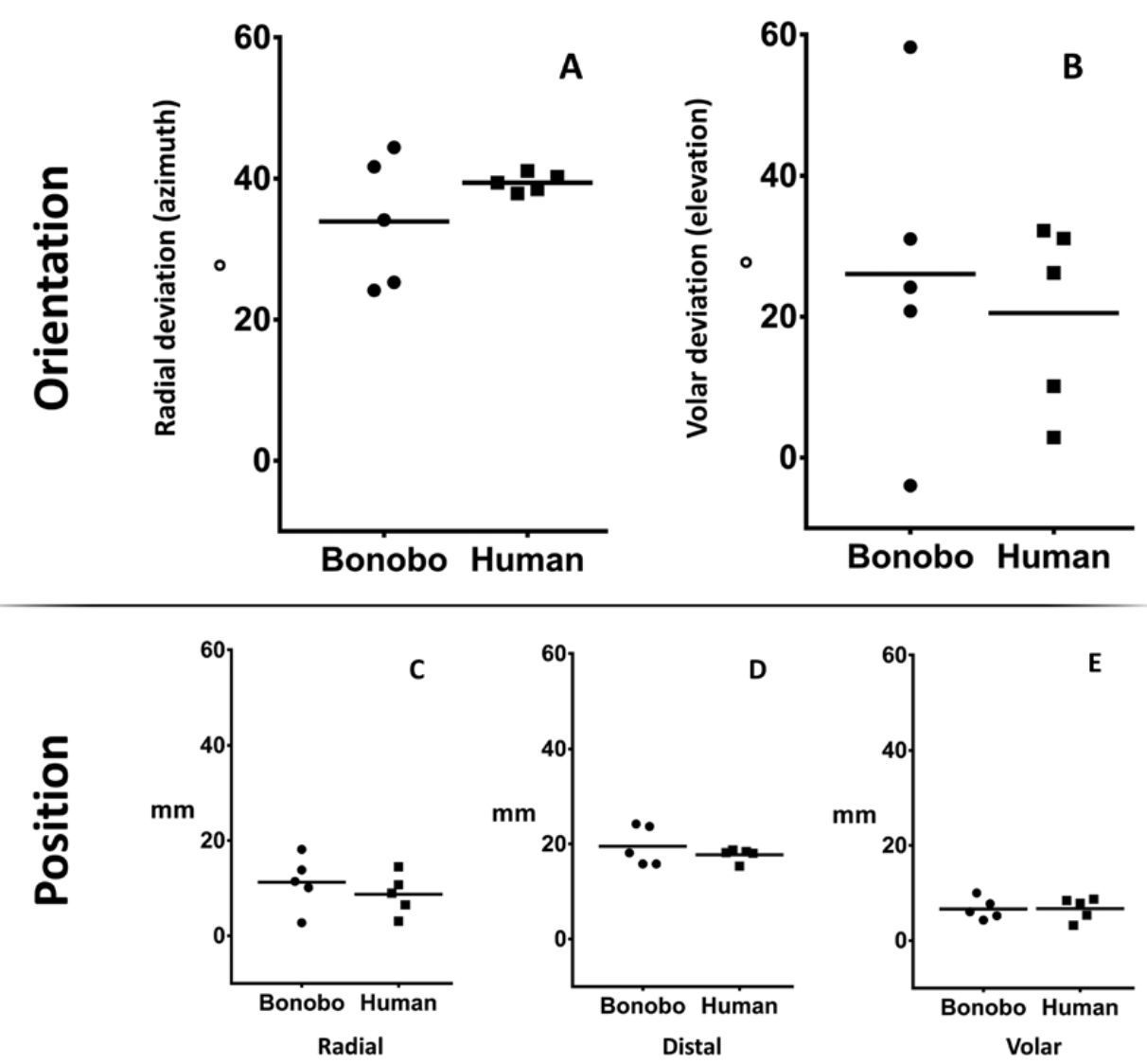

Figure 5. Orientation and position of the trapezium within the bonobo and human hand. The orientation of the trapezium distal facet is defined as the angle deviation of the trapezium normal from the proximal-distal axes of the radius, which is along the arm. Its deviation can be defined using two angles, one in the radialulnar plane, or azimuth (A), and the other in the dorsal-volar plane, elevation (B). The position of the trapezium saddle point is defined relative to the radial styloid, described in a 3D, radius-based, coordinate system with the styloid defined as origin. The trapezium saddle point is subsequently found a distance more radial (C), more distal (D) and more volar (E) to this point.

configuration mostly displays a prominent DRL that plays a significant role in TMC joint stability (Bettinger et al., 1999; D'Agostino et al., 2014) especially relative to the less prominent and weaker AOL. During thumb extension in bonobos, the relatively prominent volar AOL may restrict the range of motion compared to in humans where the relatively weak AOL cannot provide such restraint. Conversely, during flexion, the relatively weak dorso-radial ligament found in bonobos may not result in the same increase in range of motion due to additional restriction from the interaction of the first metacarpal's volar beak with the trapezium joint surface. Quantification of the $3 \mathrm{D}$ geometry of the distal trapezial facet shows that the TMC joint in both bonobos and humans is not a true saddle joint, as the dorsoventral aspect is not fully convex. Instead, the surface in this direction resembles more of a bell curve; it levels off near the periphery on both sides of the facet. Here, the distal trapezial surface captures the volar or dorsal protuberances of the first metacarpal, restricting the range of motion during flexion and extension. This restricted range of motion is seen foremost at the volar base of the facet due to the explicitly protruding volar beak of the MC1 in bonobos and humans. The triangular-shaped human volar beak is particularly interesting due to its implications for combined movements of the thumb. The apex of the volar beak limits MC1 flexion because it interacts with the volar aspect of the trapezium. At the same time, the triangular shape of the volar beak will facilitate mediolateral motions (abduction and adduction) during thumb flexion, allowing thumb opposition. In contrast, the bonobo's rounded volar beak would impede such combined motion, as noted previously in the literature (Marzke, 1992; Tocheri et al., 2003), a phenomenon that also occurs during MC1 extension in Pan and Gorilla (Rafferty, 1990).

In the mediolateral plane, the human distal trapezial articular surface has a larger radius of curvature than in bonobos, but nevertheless, the range of motion 

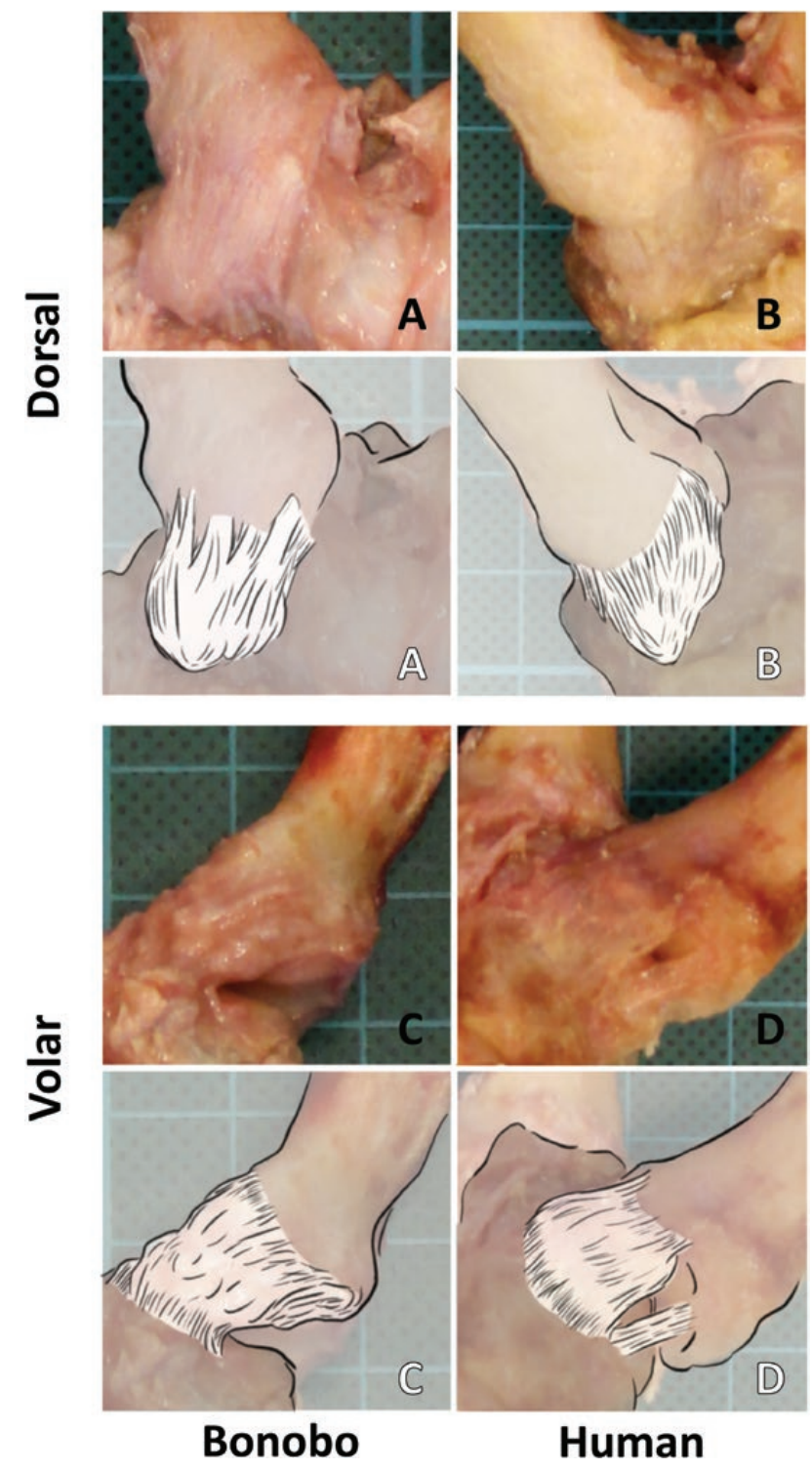

Figure 6. Pictures and illustrations of TMC joint ligament configurations in bonobos (A, C) compared to that of humans (B, D). Dorsally (A, B) we see the DRL and POL, and on the volar side (C, D) we see the AOL.

of the MC1 during thumb abduction is similar in both species. A larger radius of curvature does, however, provide a more suitable facet for combined movements (e.g. thumb opposition by the combined efforts of MC1 flexion, adduction and internal rotation), divergent from the principal axes of the trapezium, which are required to facilitate thumb opposition. The relatively 'flat' articular surface of the human trapezium has been associated with facilitating thumb opposition before (Marzke, 1992). However, a flat facet is more vulnerable to subluxation and non-human primates may, in order to stabilize the TMC against relatively high pressures involved in locomotion, require stronger ligaments, or, as described in many non-human primates, a deeper trapezial facet (Marzke et al., 2010). In our bonobo specimens, we could only discern four TMC joint ligaments (i.e. AOL, DRL, POL, IML), similar to descriptions for chimpanzees (Lewis, 1977). In humans, additional ligaments crossing the TMC joint have been described (e.g. UCL, DIML: Imaeda et al., 1993; Bettinger et al., 1999; Cardoso et al., 2009), which might suggest that the human TMC joint requires more extrinsic stabilization (e.g. due to flatter articular surfaces and/or higher joint loading).

In humans, the proximal articular facet of the MC1 has a smaller radius of curvature than the distal articular facet of the trapezium, which together mean that the mediolateral plane of the TMC joint is incongruent 
in a neutral position (Napier, 1955; D'Agostino et al., 2017b). During either abduction or adduction, however, the proximal facet on the MC1 is rotated and aligned with the distal facet on the trapezium to ensure relative congruency at the end of the movement (Napier, 1955; Rafferty, 1990). In bonobos, the more curved distal trapezial articular surface could achieve such a congruency earlier, which would limit the range of abduction-adduction of the MC1, but this was not observed in our study. Perhaps the more curved distal trapezial facet is met with a sufficiently curved MC1 articular surface that allows for the similar range of MC1 abduction-adduction as in humans. The MC1 range of motion is, however, determined not only by the radius of curvature of the articular facets, but also by the 3D geometry and surrounding soft tissue (i.e. ligaments, tendons and muscles). In addition to the TMC joint ligaments discussed above, the joint is also reinforced radially by the tendon of the abductor pollicis longus muscle, which is positioned adjacent to the DRL. This tendon (or tendons, as multiple parallel tendons occur in humans) inserts on the base of the MC1, but the muscle typically has a second tendon that inserts on the trapezium both in bonobos and in humans (van Oudenaarde, 1991; van Leeuwen et al., 2018). The second APL insertion on the trapezium is variable, and additional tendon slips spanning (and reinforcing) the TMC joint can occur (van Oudenaarde, 1991).

Comparison of our results with other studies is difficult, given that this is the first study that quantifies the kinematics of the MC1 using intact cadaveric bonobo specimens. For example, Tocheri et al. (2003) investigated the orientation of trapezial articular surfaces in extant primates and fossil hominins by studying the bony morphology of the trapezium in isolation, while our study uses in situ cadaveric specimens. Furthermore, Tocheri et al. (2003) did not quantify the curvature of the distal metacarpal facet of the trapezium. Rafferty (1990) investigated the functional morphology of the TMC joint in a large skeletal sample of extant primates, including Pan and humans, by tracing the articular facets of both the trapezium and MC1. Her results did not include any quantification of curvature but did show joint incongruity in both Pan and humans, and increased joint congruency during MC1 extension in Pan only. The single study we know of that quantified the MC1 range of motion in bonobos is the study by Rose (1992) who used dry skeletal museum specimens that were put in positions of maximum flexion-extension and abduction-adduction. The range of MC1 motion obtained during thumb abduction corresponds well for bonobos $\left[31.4 \pm 6.5^{\circ}\right.$ vs. Rose's (1992) $34.9 \pm 4.5^{\circ}$ ], but less so for humans $\left(33.0 \pm 4.5^{\circ}\right.$ vs. $\left.46.3 \pm 4.9^{\circ}\right)$. While the range of $\mathrm{MC} 1$ motion during thumb flexion corresponds well for humans $\left(38.7 \pm 3.9^{\circ}\right.$ vs. Rose's $\left.(1992) 37.6 \pm 5.3^{\circ}\right)$ and the bonobo range of MC1 motion deviates markedly from Rose's (1992) observations $\left(9.5 \pm 2.9^{\circ}\right.$ vs. $32.8 \pm 5.3^{\circ}$ ). The lack of correspondence between these studies is probably the result of methodological differences. Rose (1992) determined range of motion by aligning the articular surfaces of the trapezium and MC1 from one position to the other using dry skeletal material. Our kinematic analysis, however, was performed using intact cadaver specimens (including articular cartilage, ligaments, tendons and muscles) without being based on subjective limitations the way visual alignment does. As mentioned before, the well-developed AOL may be responsible for notable restriction of bonobo thumb extension, possibly causing overestimation of the range of MC1 motion during thumb flexion and extension in the study of Rose (1992). As Rose (1992) acknowledged, soft tissues will influence the biomechanics of the TMC joint, although it is also clear that such influences may vary. Our study shows kinematic differences between bonobos and humans that cannot be related to the $3 \mathrm{D}$ geometry of the skeletal elements. This has important implications for studies of fossil remains, as these differences are probably related to differences in soft tissue which are typically lacking in the fossil record. When studying extant apes, where both functional capabilities and morphology can be studied, it is important to investigate and understand the effects of all types of tissue in biomechanical analyses of joint systems as this can contribute to a more accurate interpretation of fossil remains.

This study provides a quantification of MC1 kinematics of bonobos in comparison to humans, and is important to help our understanding of the functional abilities of the bonobo thumb and hand. Some limitations should, however, be taken into account, such as the small sample size and the use of cadaver specimens for the bonobo analysis. Bonobos are an endangered species (Fruth et al., 2016) and specimens are difficult to obtain because the captive population is small. The small sample size does not allow us to take into account intra- and interspecific variations or to investigate sex or age differences. As such, we cannot comment on the relatively large variability displayed by the one subadult specimen. Furthermore, the use of cadaver specimens might also influence the kinematics obtained for the bonobos and might hamper the comparison with the human data set, which was obtained from healthy volunteers (active vs. passive positioning, muscle activity, etc.). This issue is, however, difficult to circumvent given that acquisition of CT scans of living bonobos is not possible.

Another limiting factor of the study is that curvatures of the distal trapezial facet were determined 
using 3D bone models reconstructed from CT scans, which excludes information on articular cartilage. Although little is known about the distribution of articular cartilage in the bonobo TMC joint, articular cartilage has been shown to be distributed nonuniformly in the human TMC joint (Dourthe et al., 2018). The actual joint curvature might therefore deviate slightly from the curvatures we measured using the CT-based bone models.

Finally, we used a standardized rig to position the bonobo and human thumb in maximum flexion-extension and maximum abduction-adduction. Although this approach ensures sufficient standardization between specimens and between humans and bonobos, we cannot ascertain that we have effectively determined the maximum range of motion in each plane. For example, the orientation of the trapezium was quantified in the position of maximum thumb extension. The amount of extension varies between specimens, resulting in wide variation in calculated elevation angle (SD of $\pm 22.3^{\circ}$ and $\pm 13.2^{\circ}$ in bonobos and humans, respectively). The other parameter, the azimuth, shows much lower variation as this was defined in the abduction-adduction plane, which was more constrained.

In our current analysis, we focus on the TMC joint and movements and mechanics of the $\mathrm{MC} 1$, but used protocol we used yields information on the entire kinematic chain of the thumb (i.e. movements of the scaphoid, trapezium and $\mathrm{MC} 1$ relative to the radius) and could be further expanded to incorporate other areas of the wrist and hand in future analysis. This study is an example of the potential of using a combination of anatomical data, for both hard and soft tissues, and biomechanical analyses.

\section{CONCLUSIONS}

The present study uses anatomical and kinematic data to provide new information on thumb mobility, specifically the trapeziometacarpal complex, of the bonobo, a hominid with a fully opposable thumb and high dexterity. We show that the anatomy and kinematics of the bonobo thumb are similar to that of humans, although we do observe differences in mechanical capabilities, articular surface geometry and ligament configuration. Most importantly, we find that humans are capable of extending their first metacarpal considerably more than bonobos, which might be related to their higher level of dexterity. Furthermore, we find kinematic differences between bonobos and humans that cannot solely be related to the 3D geometry of the skeletal elements. It appears that trapeziometacarpal joint biomechanics are influenced by both the articular surface geometry and its associated soft tissue, and both should be studied in greater detail when investigating the functional ability of the TMC joint system or any other anatomical structure. It is important to investigate and understand the effects of all types of tissue in biomechanical analyses of joint systems as this can contribute to a more accurate interpretation of fossil remains.

\section{ACKNOWLEDGEMENTS}

This paper was a contribution to a European Federation for Primatology symposium on 'What an interdisciplinary approach can tell us about the evolution of grasping and manipulation' held 21-25 August 2017 at the University of Strasbourg in France and organized by Emmanuelle Pouydebat and Ameline Bardo, the proceedings of which have been collated as a Special Issue of the Biological Journal of the Linnean Society (2019). The authors thank the Bonobo Morphology Initiative organized in January 2016 by Dr Nauwelaerts and Dr Pereboom from the Centre for Research and Conservation (Royal Zoological Society Antwerp, Belgium) that enabled access to a large part of the bonobo specimens and allowed dissection of these specimens by an international team of anatomists. We are also grateful to the different zoos which provided additional bonobo specimens: Jean-Pascal Guéry (La Vallée des Singes, Romagne, France), Christina Geiger (Zoo Frankfurt, Germany) and Martina Balz (Wilhelma Zoo, Stuttgart, Germany). Furthermore, we thank Dr Olivier Vanovermeire and Henk Lacaeyse from the Medical Imaging Department, AZ Groeninge (Kortrijk, Belgium) for performing CT scans of the specimens. Lastly, we thank reviewers Dr Tracy Kivell and Dr Biren Patel for their valuable input. Funding for this project was obtained from KU Leuven (project number: C14/16/082).

\section{AUTHOR CONTRIBUTIONS}

TvL conceived the study; EEV and TvL further designed the study; JS provided the specimens and assisted in data collection; TvL, MJMV, FDK, PDA and EEV performed the dissections; MV and TvL created the analysis; TvL and EEV analysed the data and wrote the manuscript; HvL provided feedback to the final draft; all authors reviewed and approved the article. Illustrations by TvL.

\section{REFERENCES}

Almécija S, Alba DM, Moyà-Solà S. 2012. The thumb of Miocene apes: new insights from Castell de Barberà (Catalonia, Spain). American Journal of Physical Anthropology 148: 436-450. 
Bardo A, Borel A, Meunier H, Guéry JP, Pouydebat E. 2016. Behavioral and functional strategies during tool use tasks in bonobos. American Journal of Physical Anthropology 161: 125-140.

Berger RA. 2001. The anatomy of the ligaments of the wrist and distal radioulnar joints. Clinical Orthopaedics and Related Research 383: 32-40.

Bergström P, Edlund O. 2017. Robust registration of surfaces using a refined iterative closest point algorithm with a trust region approach. Numerical Algorithms 74: 755-779.

Bergström P, Edlund O, Söderkvist I. 2011. Repeated surface registration for on-line use. The International Journal of Advanced Manufacturing Technology 54: 677-689.

Bettinger PC, Linscheid RL, Berger RA, Cooney WP $3^{\text {rd, }}$ An KN. 1999. An anatomic study of the stabilizing ligaments of the trapezium and trapeziometacarpal joint. The Journal of Hand Surgery 24: 786-798.

Breuer T, Ndoundou-Hockemba M, Fishlock V. 2005. First observation of tool use in wild gorillas. PLoS Biology 3: e380.

Butterworth G, Itakura S. 1998. Development of precision grips in chimpanzees. Developmental Science 1: 39-43.

Cardoso FN, Kim HJ, Albertotti F, Botte MJ, Resnick D, Chung CB. 2009. Imaging the ligaments of the trapeziometacarpal joint: MRI compared with MR arthrography in cadaveric specimens. American Journal of Roentgenology 192: W13-W19.

Christel M. 1993. Grasping techniques and hand preferences in Hominoidea. In: Hands of Primates. Vienna: Springer Vienna, 91-108.

Christel MI, Kitzel S, Niemitz C. 1998. How precisely do Bonobos (Pan paniscus) grasp small objects? International Journal of Primatology 19: 165-194.

Colell M, Segarra MD, Pi JS. 1995. Hand preferences in chimpanzees (Pan troglodytes), bonobos (Pan paniscus), and orangutans (Pongo pygmaeus) in food-reaching and other daily activities. International Journal of Primatology 16: 413-434.

Cooney WP III, Chao EY. 1977. Biomechanical analysis of static forces in the thumb during hand function. The Journal of Bone and Joint Surgery. American Volume 59: 27-36.

Crisco JJ, Halilaj E, Moore DC, Patel T, Weiss AP, Ladd AL. 2015. In vivo kinematics of the trapeziometacarpal joint during thumb extension-flexion and abduction-adduction. The Journal of Hand Surgery 40: 289-296.

D'Agostino P, Dourthe B, Kerkhof F, Harry Van Lenthe G, Stockmans F, Vereecke EE. 2017b. In vivo biomechanical behavior of the trapeziometacarpal joint in healthy and osteoarthritic subjects. Clinical Biomechanics 49: 119-127.

D'Agostino P, Dourthe B, Kerkhof F, Stockmans F, Vereecke EE. 2017a. In vivo kinematics of the thumb during flexion and adduction motion: evidence for a screw-home mechanism. Journal of Orthopaedic Research 35: 1556-1564.

D'Agostino P, Kerkhof FD, Shahabpour M, Moermans JP, Stockmans F, Vereecke EE. 2014. Comparison of the anatomical dimensions and mechanical properties of the dorsoradial and anterior oblique ligaments of the trapeziometacarpal joint. The Journal of Hand Surgery 39: 1098-1107.
Dourthe B, Nickmanesh R, Wilson DR, D'Agostino P, Patwa AN, Grinstaff MW, Snyder BD, Kerkhof F, van Lenthe H, Vereecke E. 2018. Assessment of healthy trapeziometacarpal cartilage properties using indentation testing and contrast-enhanced computed tomography. Clinical Biomechancics.

Feix T, Kivell TL, Pouydebat, Dollar AM. 2015. Estimating thumb-index finger precision grip and manipulation potential in extant and fossil primates. Journal of The Royal Society Interface 12: 20150176.

Fruth B, Hickey JR, André C, Furuichi T, Hart J, Hart T, Kuehl H, Maisels F, Nackoney J, Reinartz G, Sop T, Thompson J, Williamson EA. 2016. Pan paniscus (errata version published in 2016). The IUCN Red List of Threatened Species 2016: e.T15932A102331567. doi:10.2305/IUCN. UK.2016-.RLTS.T15932A17964305.en

Gumert MD, Hoong LK, Malaivijitnond S. 2011. Sex differences in the stone tool-use behavior of a wild population of burmese long-tailed macaques (Macaca fascicularis aurea). American Journal of Primatology 73: 1239-1249.

Halilaj E, Rainbow MJ, Got CJ, Moore DC, Crisco JJ. 2013. A thumb carpometacarpal joint coordinate system based on articular surface geometry. Journal of Biomechanics 46: 1031-1034.

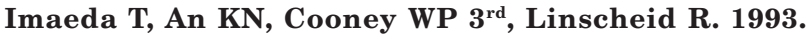
Anatomy of trapeziometacarpal ligaments. The Journal of Hand Surgery 18: 226-231.

Ingmanson EJ. 1998. Tool-using behaviour in wild Pan paniscus: social and ecological considerations. In: Russon AE, Bard KA, Parker ST, eds. Reaching into thought: the minds of the great Apes. Cambridge: Cambridge University Press, 464.

Jordan C. 1982. Object manipulation and tool-use in captive pygmy chimpanzees (Pan paniscus). Journal of Human Evolution 11: 35-39.

Kerkhof FD, Vereecke EE, Vanovermeire O, Vanhaecke J, Vanneste M, Stockmans F. 2018. Trapeziometacarpal stabilization through dorsoradial ligament reconstruction: an early post-surgery in vivo biomechanical analyses. Journal of Orthopaedic Research. doi: 10.1002/jor.24103.

Komatsu I, Lubahn JD. 2018. Anatomy and biomechanics of the thumb carpometacarpal joint. Operative Techniques in Orthopaedics 28: 1-5.

Ladd AL, Lee J, Hagert E. 2012. Macroscopic and microscopic analysis of the thumb carpometacarpal ligaments: a cadaveric study of ligament anatomy and histology. The Journal of Bone and Joint Surgery. American Volume 94: 1468-1477.

van Leeuwen T, Vanhoof MJM, Kerkhof FD, Stevens JMG, Vereecke EE. 2018. Insights into the musculature of the bonobo hand. Journal of Anatomy 233: 328-340.

Lewis OJ. 1977. Joint remodelling and the evolution of the human hand. Journal of Anatomy 123: 157-201.

Marchi D, Proctor DJ, Huston E, Nicholas CL, Fischer F. 2017. Morphological correlates of the first metacarpal proximal articular surface with manipulative capabilities in apes, humans and South African early hominins. Comptes Rendus Palevol 16: 645-654. 
Marzke MW. 1971. Origin of the human hand. American Journal of Physical Anthropology 34: 61-84.

Marzke MW. 1992. Evolutionary development of the human thumb. Hand Clinics 8: 1-8.

Marzke MW. 1997. Precision grips, hand morphology, and tools. American Journal of Physical Anthropology 102: 91-110.

Marzke MW, Marchant LF, McGrew WC, Reece SP. 2015. Grips and hand movements of chimpanzees during feeding in Mahale Mountains National Park, Tanzania. American Journal of Physical Anthropology 156: 317-326.

Marzke MW, Tocheri MW, Steinberg B, Femiani JD, Reece SP, Linscheid RL, Orr CM, Marzke RF. 2010. Comparative 3D quantitative analyses of trapeziometacarpal joint surface curvatures among living catarrhines and fossil hominins. American Journal of Physical Anthropology 141: 38-51.

Napier JR. 1952. The attachments and function of the abductor pollicis brevis. Journal of Anatomy 86: 335-341.

Napier JR. 1955. The form and function of the carpo-metacarpal joint of the thumb. Journal of Anatomy 89: 362-369.

Napier JR. 1960. Studies of the hands of living primates. Proceedings of the Zoological Society of London 134: 647-657.

Neufuss J, Humle T, Cremaschi A, Kivell TL. 2017. Nutcracking behaviour in wild-born, rehabilitated bonobos (Pan paniscus): a comprehensive study of hand-preference, hand grips and efficiency. American Journal of Primatology 79: $1-16$.

Orr CM, Leventhal EL, Chivers SF, Marzke MW, Wolfe SW, Crisco JJ. 2010. Studying primate carpal kinematics in three dimensions using a computed-tomography-based markerless registration method. Anatomical Record 293: 692-709.

van Oudenaarde E. 1991. Structure and function of the abductor pollicis longus muscle. Journal of Anatomy 174: 221-227.

Pouydebat E, Gorce P, Coppens Y, Bels V. 2009. Biomechanical study of grasping according to the volume of the object: human versus non-human primates. Journal of Biomechanics 42: 266-272.

Rafferty KL. 1990. The functional and phylogenetic significance of the carpometacarpal joint of the thumb in anthropoid primates. New York University.

Rose MD. 1992. Kinematics of the trapezium-1st metacarpal joint in extant anthropoids and Miocene hominoids. Journal of Human Evolution 22: 255-266.

Samuel DS, Nauwelaerts S, Stevens JMG, Kivell TL. 2018. Hand pressures during arboreal locomotion in captive bonobos (Pan paniscus). The Journal of Experimental Biology 221. doi: 10.1242/jeb.170910.
Shrewsbury M. 2003. Pollical oblique ligament in humans and non-human primates. Journal of Anatomy 202: 397-407.

Skinner MM, Stephens NB, Tsegai ZJ, Foote AC, Nguyen NH, Gross T, Pahr DH, Hublin JJ, Kivell TL. 2015. Human evolution. Human-like hand use in Australopithecus africanus. Science 347: 395-399.

Spinozzi G, Truppa V, Laganà T. 2004. Grasping behavior in tufted capuchin monkeys (Cebus apella): grip types and manual laterality for picking up a small food item. American Journal of Physical Anthropology 125: 30-41.

Takeshita H, Walraven V. 1996. A comparative study of the variety and complexity of object manipulation in captive chimpanzees (Pan troglodytes) and bonobos (Pan paniscus). Primates 37: 423-441.

Tocheri MW, Marzke MW, Liu D, Bae M, Jones GP, Williams RC, Razdan A. 2003. Functional capabilities of modern and fossil hominid hands: three-dimensional analysis of trapezia. American Journal of Physical Anthropology 122: 101-112.

Tocheri MW, Orr CM, Jacofsky MC, Marzke MW. 2008. The evolutionary history of the hominin hand since the last common ancestor of Pan and Homo. Journal of Anatomy 212: 544-562.

Tocheri MW, Razdan A, Williams RC, Marzke MW. 2005. A $3 \mathrm{D}$ quantitative comparison of trapezium and trapezoid relative articular and nonarticular surface areas in modern humans and great apes. Journal of Human Evolution 49: 570-586.

Toth N, Schick KD, Savage-Rumbaugh ES, Sevcik RA, Rumbaugh DM. 1993. Pan the tool-maker: investigations into the stone tool-making and tool-using capabilities of a Bonobo (Pan paniscus). Journal of Archaeological Science 20: 81-91.

Tuttle RH. 1967. Knuckle-walking and the evolution of hominoid hands. American Journal of Physical Anthropology 26: 171-206.

Wu G, Cavanagh PR. 1995. ISB recommendations for standardization in the reporting of kinematic data. Journal of Biomechanics 28: 1257-1261.

Wu G, van der Helm FC, Veeger HE, Makhsous M, Van Roy P, Anglin C, Nagels J, Karduna AR, McQuade K, Wang X, Werner FW, Buchholz B; International Society of Biomechanics. 2005. ISB recommendation on definitions of joint coordinate systems of various joints for the reporting of human joint motion-Part II: shoulder, elbow, wrist and hand. Journal of Biomechanics 38: 981-992.

Wunderlich RE, Jungers WL. 2009. Manual digital pressures during knuckle-walking in chimpanzees (Pan troglodytes). American Journal of Physical Anthropology 139: 394-403. 